\title{
Study of Fetal Neurodevelopment in Multiple Pregnancies
}

\section{${ }^{1}$ Ingrid Marton, ${ }^{2}$ Asim Kurjak, ${ }^{3}$ Guillermo Azumendi, ${ }^{4}$ Madeeha Al-Noobi, ${ }^{5}$ Sejfulah Perva}

${ }^{1,2}$ Department of Obstetrics and Gynecology, Medical School University of Zagreb, Sveti Duh Hospital, Zagreb, Croatia ${ }^{3}$ Clinica Gutenberg, Malaga, Spain

${ }^{4}$ Qatar Armed Forces, Medical Services, Doha, Qatar

${ }^{5}$ Department of Obstetrics and Gynecology, Women's Hospital, Hamad Medical Corporation, Doha, Qatar

Correspondence: Ingrid Marton, Department of Obstetrics and Gynecology, Medical School University of Zagreb, Sveti Duh Hospital, Sveti Duh 64, Zagreb, Croatia, e-mail: ingrid.marton@zg.t-com.hr

\begin{abstract}
According to many reports multiple pregnancies compared to singelton have a 5-10 fold increased risk for CP and some of increased risk is due to the risk of preterm delivery, no mater whether, it mono or dizygotic conception. In those cases CP is most probably complication of peripartal periventricular hemorrhage or leukomalacia. Known fact about twins is that the lower is the gestational age at the time of delivery, the probability that they are monozygotic is greater. Monochorionic multiple pregnancies and feto-fetal hemodynamic transfusion instability are leading to cerebral ischemia. For all of mentionable reasons monozygotic multiple pregnancies have a greater risk of cerebral impairment.

$\mathrm{CP}$ in an apparently singleton pregnancy could be associated with a "vanishing" twin phenomenon. There are reports that some of the congenital anomalies in apparently singleton pregnancies may be associated with the unrecognized or unrecorded loss of a co-twin.

It has been established that ART conceptions are at a greater risk of CP because of the greater risk of preterm delivery. There have been many attempts to minimize CP prevalence by reduction of number of fetuses in a multifetal ART pregnancy but without affect, most probably because the majority of ART conceptions are dizygotic.
\end{abstract}

Keywords: Cerebral palsy, assisted reproduction techniques, multiple pregnancies, ultrasound, fetal neurology.

\section{INTRODUCTION}

The use of assisted reproduction techniques (ART) has resulted in significant increase in the incidence of multiple pregnancies throughout the world. The nature of twinning often places twins at higher risk of perinatal and neonatal morbidity and mortality, including developmental and behavioral problems.

The primary modality for diagnosing and evaluating multiple pregnancies is still two-dimensional sonography (2D) (Figs 1 and 2). However, 2D sonography can be soon overcamed by three-dimensional (3D) and four-dimensional (4D) sonography. These techniques are complementary to 2D sonography providing with additional information that assists in the clinical management (Figs 3 to $4 \mathrm{~B}$ ).

Nowadays the most frequently used technique for detection and evaluation of intertwin contacts is fourdimensional (4D) sonography, because it allows simultaneous visualization of both fetuses and assessment

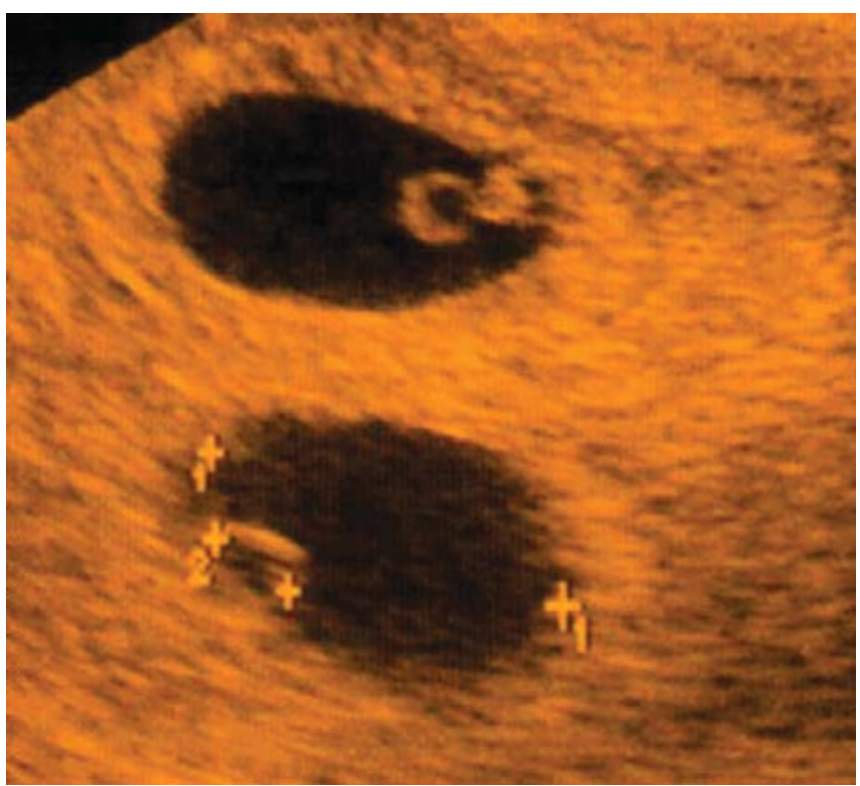

Fig. 1: The first visible structure of dizygotic and diamniotic twins by 2D US. Note the number of gestational sac and yolk sac 


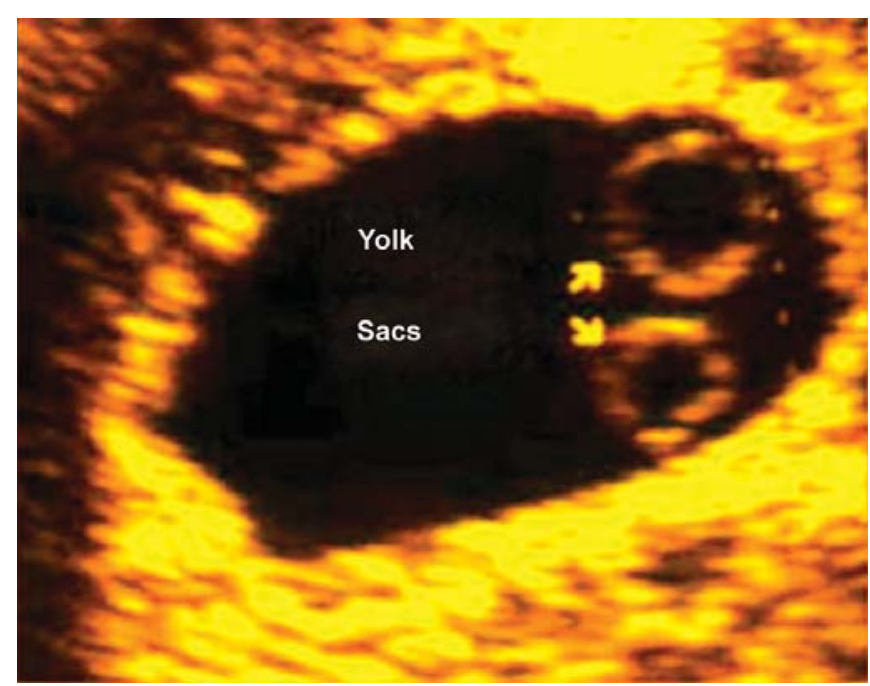

Fig. 2: The separated yolk sacs are demonstrated by 2D US in early diagnosis of monozygotic twins

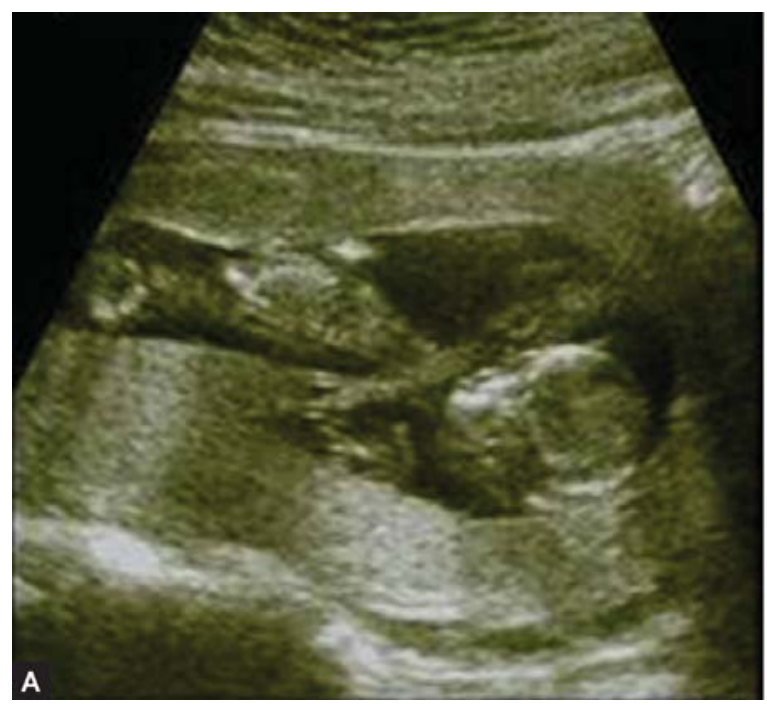

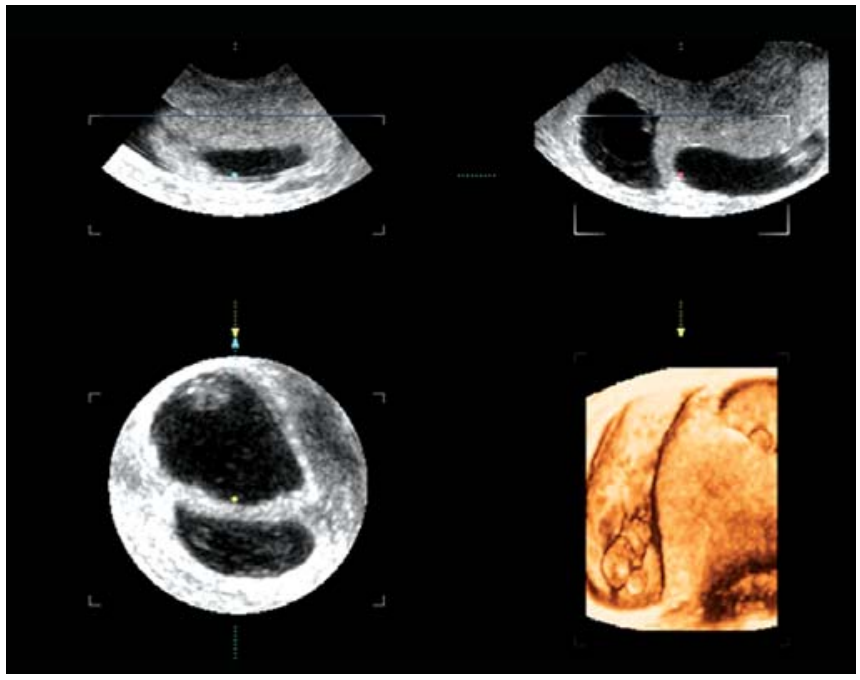

Fig. 3: Multiplanar and surface rendering of a twin pregnancy at 7 weeks gestation demonstrates the advantages of $3 D$ US in the evaluation of early multiple pregnancies

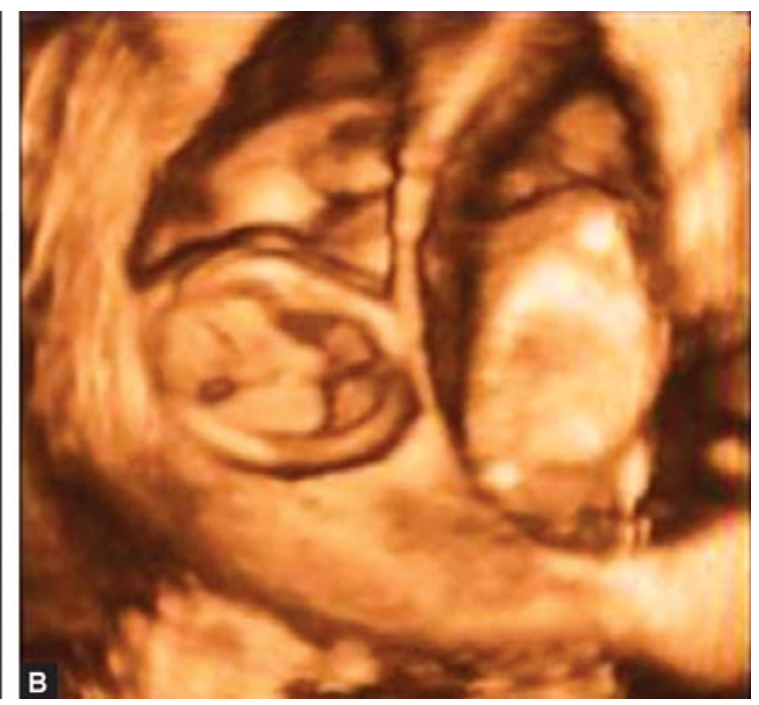

Figs 4A and B: Comparison between sectional 2D image $(A)$ and $3 D$ spatial imaging $(B)$. The advantage of 3D spatial visualization in early pregnancy includes improved visualization of both fetuses and their gestational sacs

of their motor activity. The main benefits of 4D sonography include: accurate recognition of an isolated motor activity of a single fetus; distinguishing between spontaneous and stimulated motor activity; and spatial visualization of the intertwin area (Fig. 5). ${ }^{1}$

Fetal activity such as kicking has been used as a sign of fetal viability from ancient times and was found that fetal motor activity is present for some period before the mother feels it. One of the first to describe fetal activity using ultrasound was Reinold, stressing the spontaneous character of early prenatal movements. ${ }^{2}$ Furthermore, de Vries and colleagues described types of fetal activity according to their onset in singleton pregnancy. ${ }^{3}$ Hooker showed that the embryo responds to a stimulus. ${ }^{4}$ The embryos used for this purpose originated from pregnancies terminated by hysterectomy. They were maintained in an isotonic bath. The discovery that the fetus responds to a tactile stimulus created a hypothesis that the motor activity of a twin could be either spontaneous or induced by the co-twin. Dominant behavioral pattern in singleton pregnancy is endogenous activity. The mother's movements such as walking or running can initiate fetal motor activity. On the other hand, in multiples two types of fetal activity are present: endogenous-spontaneous motor activity and motor activity that is a reaction to an exogenous stimulus. This hypothesis was confirmed in vivo by Arabin and colleagues, who 


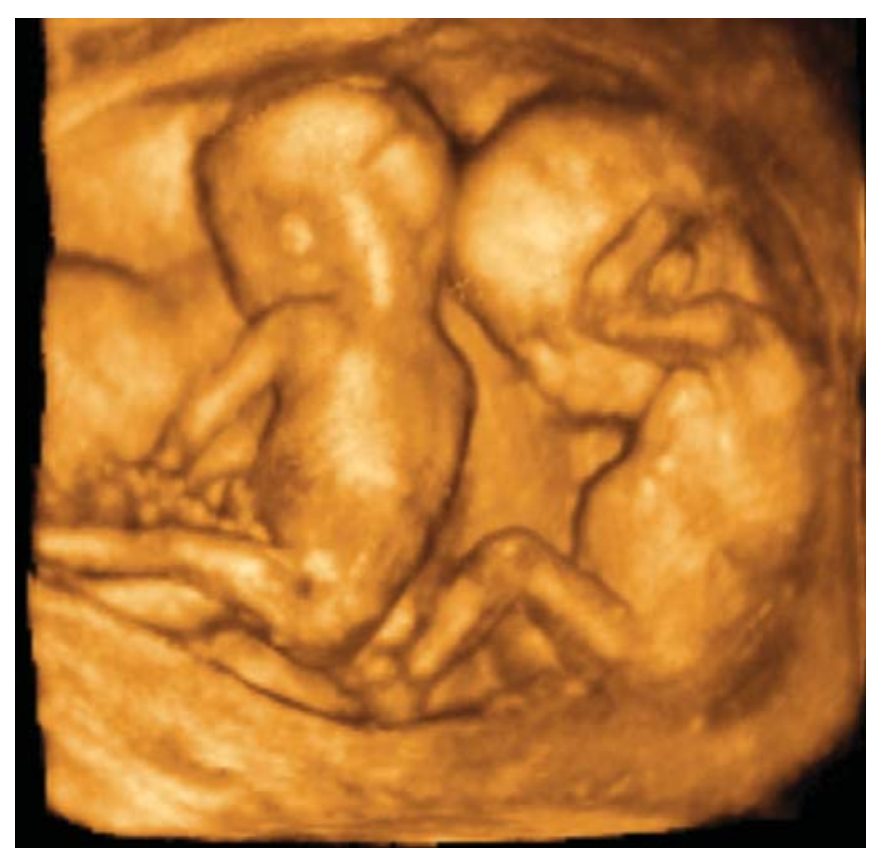

Fig. 5: A 3D sonography of a monochorionic twin pregnancy at 14 weeks gestation. Note the intertwine area that can be easily assessed

reported an embryonic reaction to a tactile stimulus between 8 and 9 weeks of gestation. ${ }^{5}$ In advanced pregnancy, with the use of real-time 2D sonography, according to the part of body, four types of intertwin contacts were recognized (contact with the head, contact with the body, contact with the hand and contact with the leg). ${ }^{5}$ According to the speed of initiation and reaction, four combinations of interactions were recognized: slow or fast initiations and slow or fast reactions. Slow initiation was equal to a tactile stimulus, and fast initiation to a kick stimulus.

\section{NEUROLOGICAL DEVELOPMENT IN MULTIPLE PREGNANCIES}

One of the first to describe fetal activity using ultrasound was Reinold, who stressed the spontaneous character of early prenatal movements. ${ }^{6}$ There are two types of motor activity in multiple pregnancies: spontaneous and stimulated. Spontaneous motoric activity is defined as each embryonic or fetal activity which not evoked by internal or external stimuli. ${ }^{7}$ Stimulated activity is evoked by intertwin contacts. Spontaneous motoric activity proceeds to stimulated motoric activity. The effect of prenatal reactions evoked by internal stimulus in twinning phenomenon due to intertwin contacts on the neurologic maturation was in the focus of interest of the systematic research initiated by Arabin. ${ }^{5}$ They used realtime 2D sonography for detection and evaluation of the intertwin contacts. Due to sectional imaging, simultaneous visualization of both fetuses and assessment of their motor activity was impossible. The intertwin area is tomogramically visualized and some of intertwin contacts are overlooked. Therefore, using this method distinguishing between spontaneous and stimulated motor activity is very difficult and sometimes impossible. If one performs 3D sonography complete anatomy of more fetuses and embryos can be visualized simultaneously. In spite achieved progress this technology is not suitable for behavioral research because the images are static and movements in the focus of interest for behavioral studies could cause significant artifacts in visualization.

Our team found out that movement activity of each fetus in twin or multiple pregnancy can be easily determined by 4D sonography in the first and early second trimester. ${ }^{7,8}$ For the first time we can see that one twin is active whereas, co-twin or co-triplets are active or not. Simultaneous visualization of motoric activity of each fetus enables study of their isolated motoric activity.

The first who defined the intertwin contacts was Arabin. ${ }^{5}$ Among them, complex interactions are recognized but complete definition of this phenomenon could not be achieved due to limitations of real-time 2D sonography. According to this group of authors those movement patterns are consisted of initiations and reactions of both twins which are sometimes difficult to distinguish and last longer than a few seconds. Complex parts of these interactions could be analyzed using 4D sonograhy for the first time. Furthermore, 4D sonography could be useful in evaluation of the altered motoric development such as in pathologic pregnancy.

Hypothesis that movements of one twin stimulate the other to move was introduced by Sadovsky. ${ }^{9}$ Ferrari and co-workers came to different conclusion. According to their observations intertwin contacts have been supposed to cause increased rates of simultaneous twin activities in early pregnancy. ${ }^{10}$ Arabin and co-workers used video documentation for continues observation of intertwin activity. ${ }^{5}$ The first interhuman contacts were determined (action and reaction within a second of interaction). Using the speed of initiations this group found slow initiations followed by slow or fast reactions and fast initiations followed by slow or fast reactions. ${ }^{5,7}$

\section{DIFFERENT SONOGRAPHIC METHODS FOR ASSESSMENT OF FETAL BEHAVIOR}

Total fetal activity is called fetal behavior. Some activities can be seen by ultrasound. 


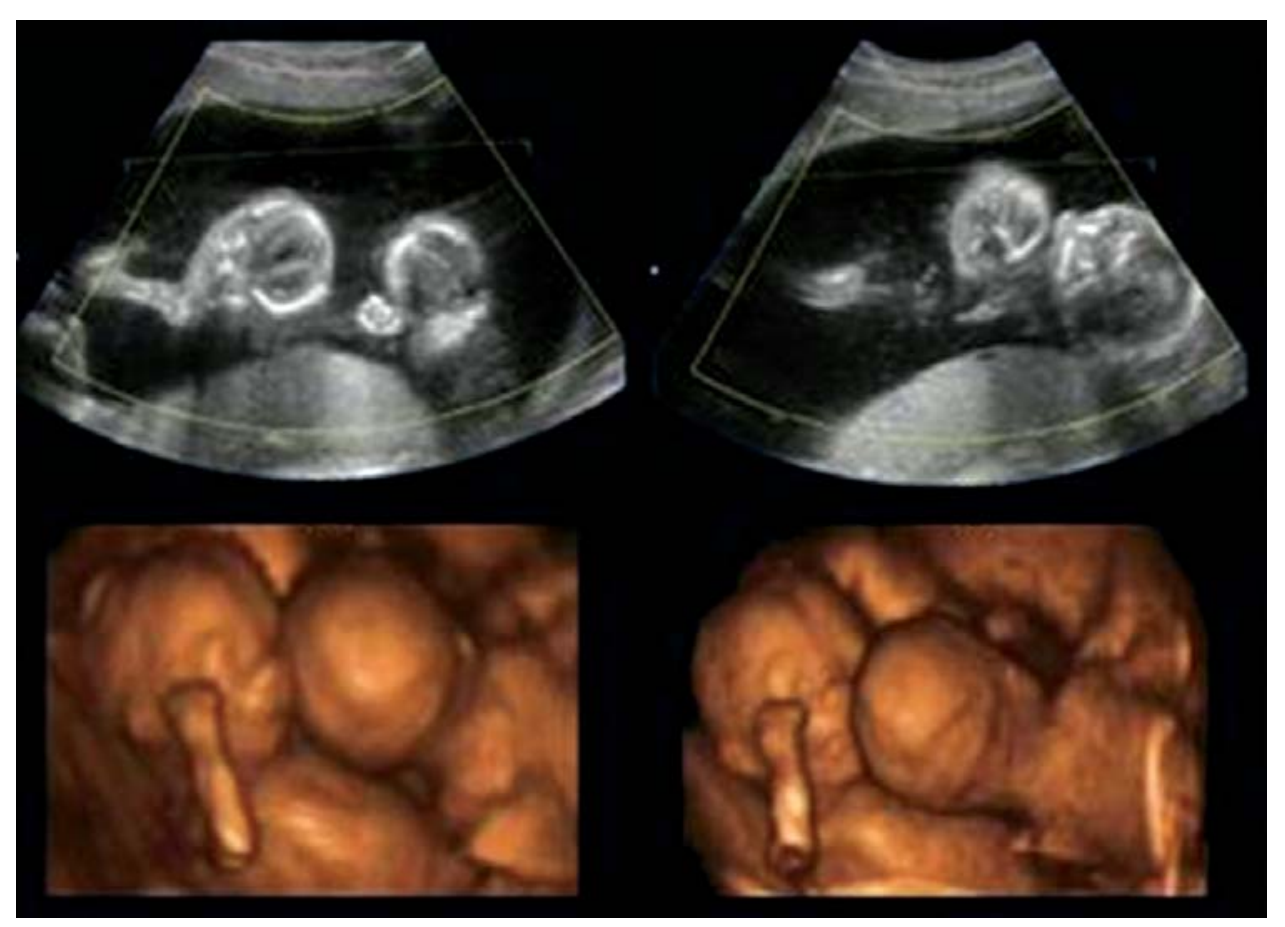

Fig. 6: Comparison between real-time 2D (upper) and 4D US (below). Those two techniques are used for evaluation of fetal behavior. Hand-to-head contact together with head-to-head intertwin contact can be recognized
Introduction of ultrasound has revolutionized the antenatal diagnosis of multiple pregnancies. Thirty years ago, twins were most often overlooked, whereas, triplets were diagnosed only after the delivery of the second infant. After introduction of ultrasound into routine prenatal care the correct diagnosis of multiple pregnancy became a part of everyday clinical practice.

Indeed, two-dimensional sonography (2D) is still the primary modality for diagnosing and evaluating multiple pregnancy. New techniques (3D and 4D) should be accepted as complementary to 2D sonography because additional information that assists in the clinical management could sometimes be provided (Fig. 6).

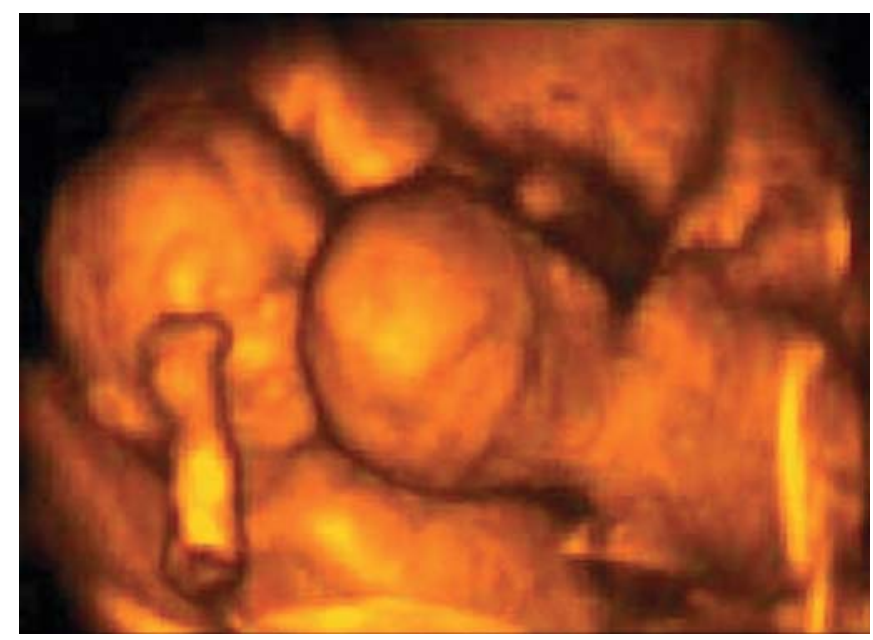

Fig. 7: Note the 3D surface rendering that demonstrates intertwin contact in a case of monochorionic-monoamniotic twin pregnancy
Modern 4D machines are capable of performing spatial imaging in near real-time providing up to 28 frames per second. ${ }^{11} 4 \mathrm{D}$ in multiple pregnancies is being used for detection and evaluation of intertwin contacts, as it allows simultaneous visualization of both fetuses and assessment of their motor activity. The main benefits of 4D sonography include: accurate recognition of an isolated motor activity of a single fetus; distinguishing between spontaneous and stimulated motor activity; and spatial visualization of the intertwin area (Figs 7 to 9). ${ }^{1}$

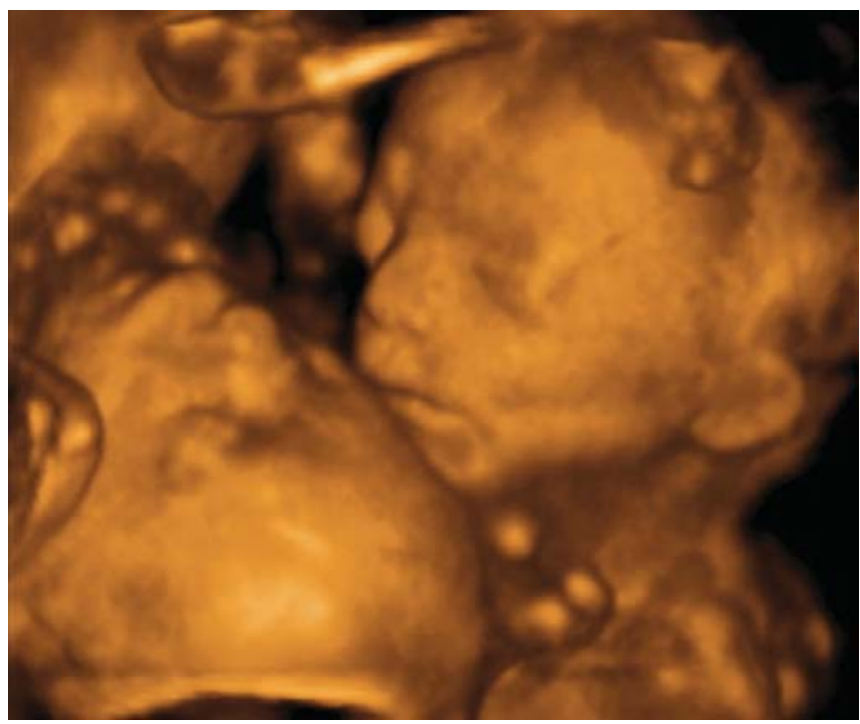

Fig. 8: Intertwin head-to-head contact at 21 weeks of gestation shown by 3D US 


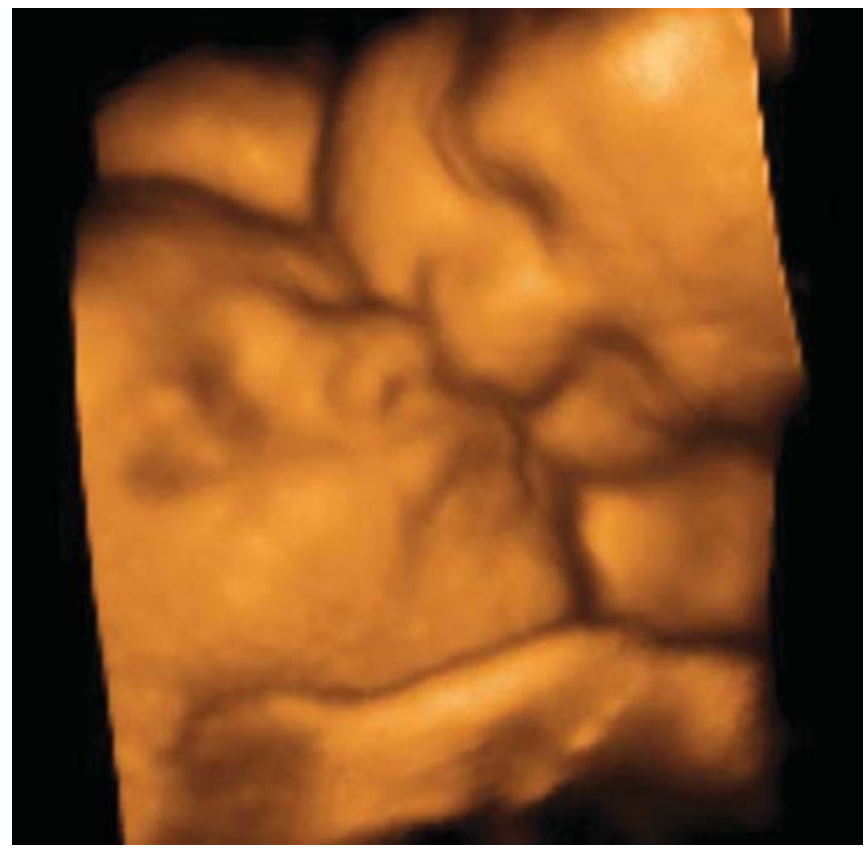

Fig. 9: Close intertwin face-to-face contact shown by 3D US

Numerous improvements in data processing capacity during the past years made it possible to overcome the previous limitations of 3D/4D ultrasound. At present, no fetal position makes it impossible to obtain a desired section and the only limitation for visualization of a desired structure is the amount of adjacent amniotic fluid. ${ }^{12}$

\section{ASSESSMENT OF FETAL BEHAVIOR BY 4D SONOGRAPHY}

The effect of prenatal reactions evoked by internal stimulus in twinning phenomenon due to intertwin contacts on the neurologic maturation was in the focus of interest of the systematic research first initiated by Arabin. ${ }^{13}$ They used real-time 2D sonography for detection and evaluation of the intertwin contacts. Due to sectional imaging, simultaneous visualization of both fetuses and assessment of their motor activity was impossible. Therefore, motor activity of a single fetus was possible to assess and unfortunately only to a limited extent. Similarly, the intertwin area is tomogramically visualized and some of intertwin contacts are overlooked. Therefore, using this method distinguishing between spontaneous and stimulated motor activity is very difficult and sometimes impossible.

From our team Vecek and colleagues. ${ }^{8}$ found that movement activity of each fetus in twin or multiple pregnancies can be successfully determined by $4 \mathrm{D}$ sonography in the first and early second trimester. For the

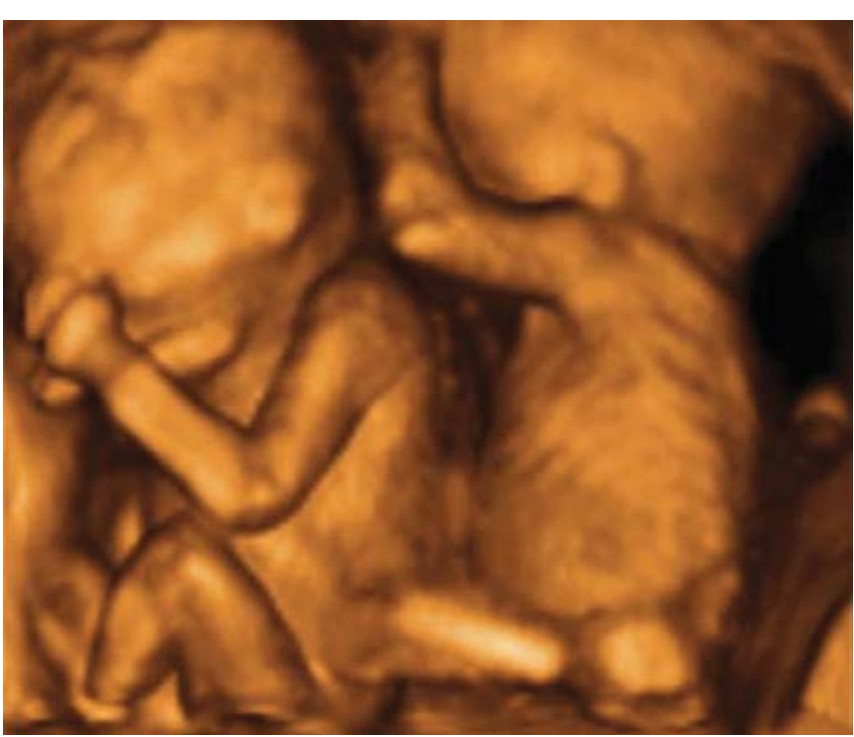

Fig. 10: Note different movements in a twin pregnancy at 21 weeks of gestation

first time it was possible to observe that one twin is active whereas, co-twin or co-triplets are active or not. Simultaneous visualization of the entire anatomy (head, body and extremities) of two or more fetuses along with their movements allows the characterization of the type of movement, isolated movements of each fetus, as well as intertwin contacts and interactions (Fig.10).

Different types of movements such as: isolated movement of each twin or high order multiples, intertwin contacts and interactions could be recognized without difficulties by 4D sonography (Fig. 11).

Intertwin contacts were first defined by Arabin who differentiated the following types of inter-twin contacts and determined the gestational age of their onset: first reach and touch (first evidence of touch between twins), first reaction (first movement towards the touch of the co-twin), slow and fast arm, leg, head or body contacts (action and reaction based on the initiating part of the body), mouth contacts and complex interactions. ${ }^{5,14,15}$ Apart from the descriptive definitions, they also classified the speed of actions and reactions as slow initiations followed by slow or fast reactions and fast initiations followed by slow or fast reactions. ${ }^{5,14}$ Inter-twin contacts may be explained by the onset of movements whereby incidental touches in utero cannot be avoided and can be defined as early reflexes. Furthermore, the initiating and reacting body parts may be randomly involved. However, Piontelli and colleagues state that the existence of intrapair stimulation would indicate merely the functioning of fetal tactile and propriceptive sensibility. ${ }^{15}$ 

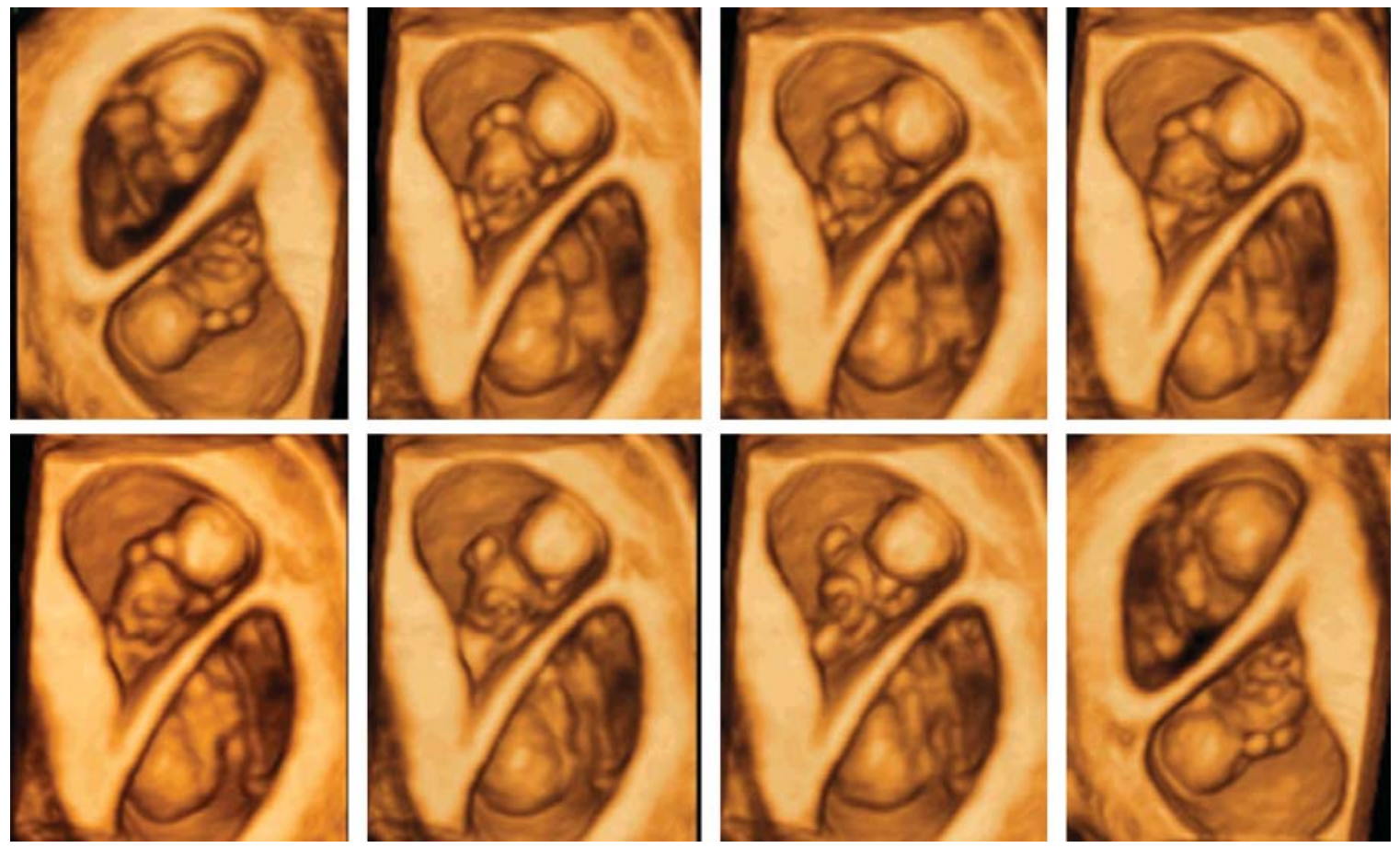

Fig. 11: A 4D US sequence of a BA/BC twin pregnancy at 10 weeks of gestation. Both twins are active, but without intertwin contact

Some authores stressed out that the existence of intrapair stimulation should not be taken to mean, as suggested by Arabin et al. that fetuses are having complex intrapair intercourses, but simply that intrapair stimulation exists constantly from late first trimester and is an active part of its intrauterine environment. ${ }^{5,14}$ The conclusion worth to be quoted is the one from Sadovsky: "The movements of one twin stimulate the other to move". ${ }^{9}$ Ferrari and coworkers came to different conclusion; according to their observations intertwin contacts have been supposed to cause increased rates of simultaneous twin activities in early pregnancy. ${ }^{10}$

According to Arabin and co-workers the distribution of the periods with or without fetal movements demonstrates the high percentage of active periods (mean around 80\%) in twins compared to singletons, which might be the result of inter-twin stimulations. ${ }^{14}$ The observation period was 26-36 weeks, and a 3-min window was used. In contrast Sadovsky observed twin pregnancies between 33 and 39 weeks. The observed fetal movements around $75 \%$ were independent of the movements of the co-twin. ${ }^{9}$ Another group found that each twin, regardless of zygosity, showed individualized behavioral styles. One twin was found to be "dominant" in the sense of greater activity, but less reactive, possibly due to the fewer stimuli being generated by its cotwin. Monozygotic twins, as opposed to dizygotic, showed greater similarities in activity and reactivity levels, but were never behaviorally identical and decreased in likeness with increasing age. Their data suggest that so-called identical twins are not behaviorally identical from early in pregnancy. ${ }^{15}$

Study conducted by Sadovsky demonstrated the significance of membrane status and its direct impact on the onset and quantity of inter-twin contacts: in MC/MA twins contacts occur earlier than in MC/DA and BC twins and even more to DC twins, which may be interpreted with the smaller distance between the embryos. ${ }^{9}$ Intrapair stimulation before the 11th week of gestation can be considered a rather exceptional event and was noted only in monochorionic twins. From 12 weeks onward intrapair stimulation becomes a progressively more frequent event both in monochorionic and dichorionic pregnancies. However, these results are from a 2D study, and thus movements occurring outside the plane of scan could have been missed. This is particularly relevant after 13 weeks of gestational age when simultaneous visualization of both fetal bodies is no longer possible. ${ }^{15}$

$4 \mathrm{D}$ sonography is very useful in evaluation of the altered motor development such as in pathologic pregnancy. ${ }^{11}$ The delay in activity pattern is described in twins with tripoidy $\mathrm{XXX}$, and some activities such as yawning and stretching are even not present. ${ }^{16}$ This observation has particular importance in dizygotic twinning. 
Table 1: Classification of cerebral palsy

Anatomical site of brain lesion

Cerebral cortex, pyramidal tract, extra pyramidal tract or cerebellum and its connections

Clinical symptoms and signs

Topographical involvement of extremities

Degree of muscle tone

Timing of the presumed insult
Spasticity, dyskinesia or ataxia

Quadriplegia, displegia, double hemiplegia Isotonic, hypotonic or hypertonic Prenatal, intrapartal, postnatal

\section{MULTIPLE PREGNANCY AND CEREBRAL PALSY}

Cerebral palsy (CP) is the most common severe physical disability in childhood affecting about 2 per 1000 live-born children (Table 1). In spite of the magnitude and severity of the problem, the routine date sources are poor in terms of epidemiology of this condition. Multiple pregnancies are known to be at higher risk for CP. The prevalence of the disease is significantly higher in twins comparing to singletons (five to ten fold increased risk), although the type of CP differs as well as the etiology of CP in twins and singletons. ${ }^{17,18}$ Data clearly indicate that the higher the number of fetuses, the greater is the prevalence of CP. When comparing twin with singleton births the relative risk of CP was greatest and significant only for twins delivered at $\geq 37$ weeks ' gestation. Brain damage of the survivor following single fetal demise is almost exclusively seen in monochorionic twins in which intertwin transplacentar vascular connections are always found, and on these grounds the "embolic" and the "ischemic" theory were found. ${ }^{19}$

Multiple pregnancies are almost by definition related to different complications such as: growth restriction, birth defects and intrapartal complications. The main reason for CP seems to be associated with preterm birth or the antenatal death of a co-twin or co-triplet (Figs 12 and 13). ${ }^{20-22}$

The death of one twin surly affects the neurological development of the survivor (Figs 14 and 15). The liveborn co-twin of a fetus dying in utero has a $20 \%$ overall risk of cerebral impairment. The gestational-age-specific prevalence of CP after fetal death of the co-twin is much higher than the reported for the general twin population. ${ }^{11}$ At particular risk are monozygotic twins who have poorer survival rates compared to dizygotic twins. The estimated prevalence of CP in monozygotic twins is 106 per 1000 infant survivors, while for dizygotic 29 per 1000 infant survivors. ${ }^{19}$ Other researchers stressed out that twins have approximately 4-fold increased risk of CP compared with

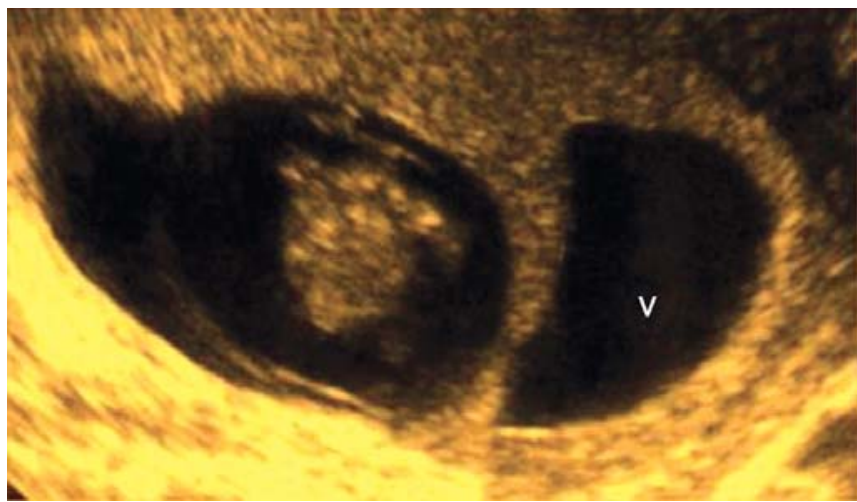

Fig. 12: A 2D transvaginal scan of dichorion twin complicated by a vanished twin (right image)

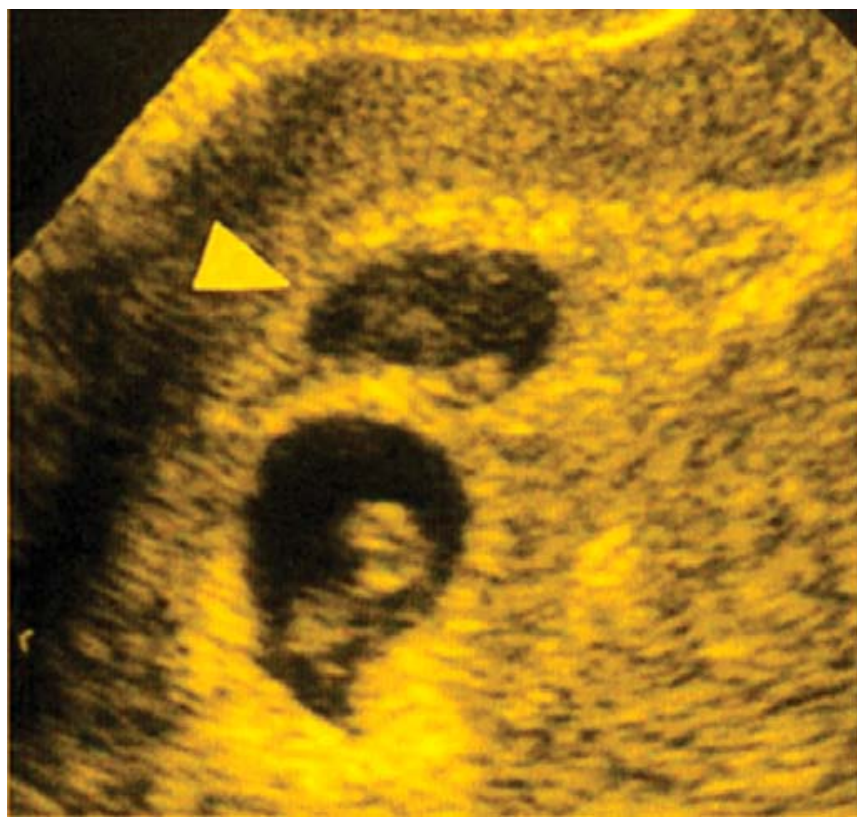

Fig. 13: A 2D US showing vanishing twin phenomenon in dichorionic twin pregnancy

singletons. However, at birth weight $<2500$ gm, twins did better than singletons. ${ }^{16}$ Approximately $70 \%$ of pregnancies with two sacs/embryos continue as twin gestations, while in contrast only about $50 \%$ of pregnancies with three sacs/ embryos will continue as triplets, and only about $38 \%$ of pregnancies with four sacs/embryos will continue as quadruplets. ${ }^{23}$ Authors suggest that vanishing rates are more than twice as high in monochorionic compard with dichorionic twin gestations (50\% vs 21\%). ${ }^{24}$ The vanishing twin phenomenon was first recognized by ultrasound; which in conjunction with the observation that normal birth weight twins are at increased risk of CP compared with singletons, led to the hypothesis that cerebral palsy of unknown etiology in singleton pregnancies may be attributed to the early loss 

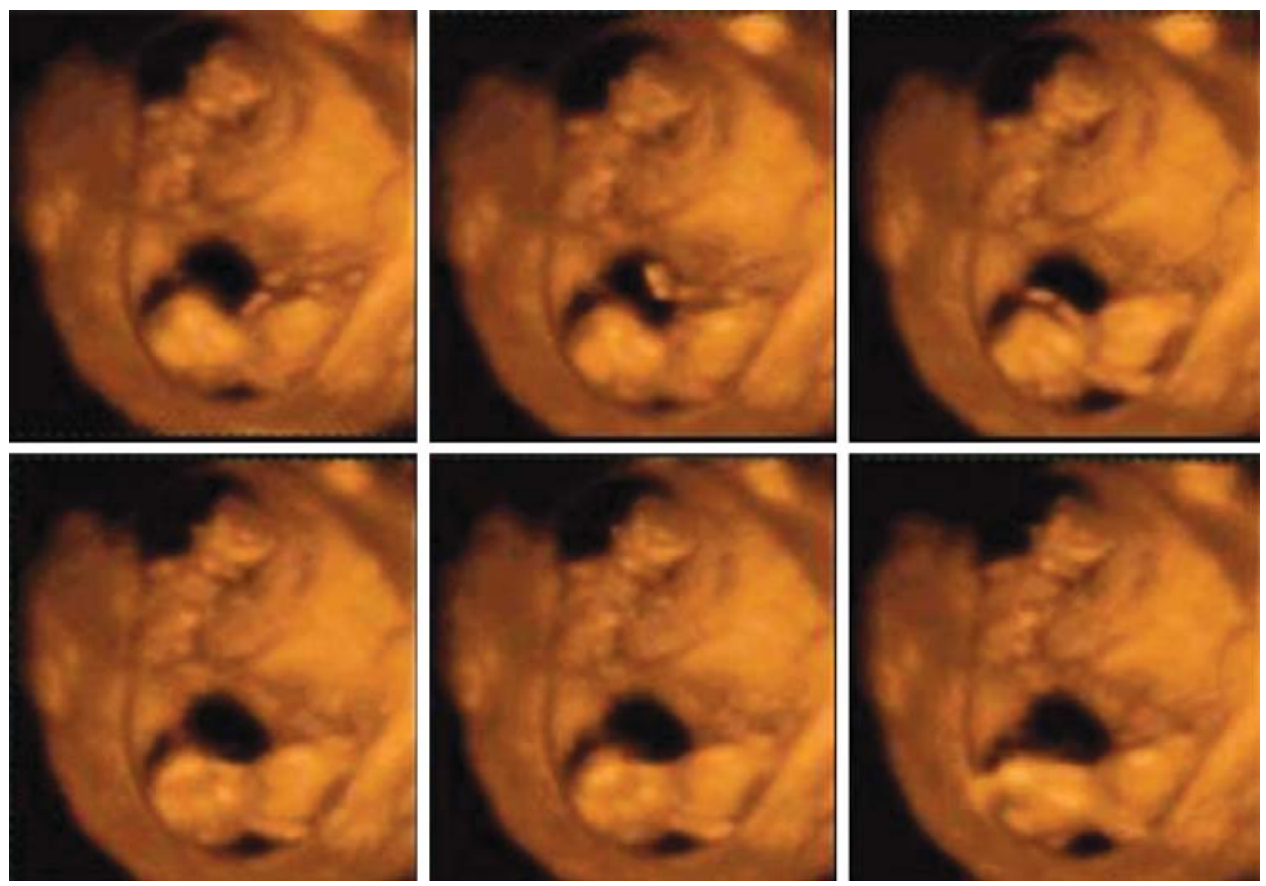

Fig. 14: A 4D sequence showing missed triplet and isolated motoric activity of only one fetus. The activity of only one twin can be recognized. Moreover, because of spatial imaging even type of movement can be recognized and that is isolated hand to fluid movement of the first triplet
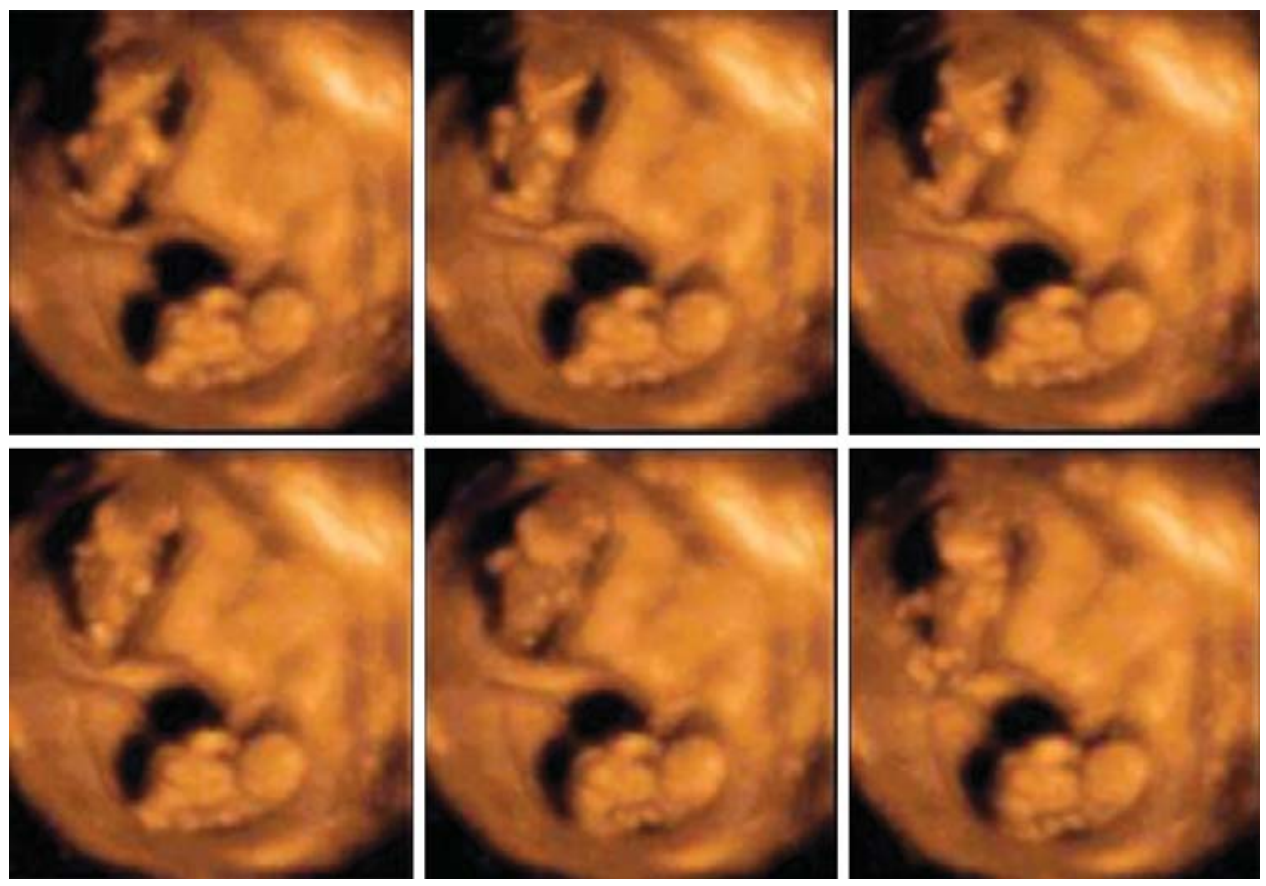

Fig. 15: A 4D scan of missed triplet at 13th weeks of gestation. Activity of only one twin can be recognized

of a previously unrecognized monochorionic twin. ${ }^{25}$ Accordingly the survivor's neurological development is impaired throughout pregnancy, with spastic $\mathrm{CP}$ as a resulting clinical manifestation. ${ }^{26}$ Data are not consistently supportive with this concept and state that the vanishing twin syndrome is unlikely to account for a high proportion of cases of CP. Against such a concept is a lack of reported cases following multifetal pregnancy reduction, a procedure that is not unlike the vanishing twin phenomenon. There was an attempt to determine whether iatrogenic fetal reduction increases the prevalence of $\mathrm{CP}$, and results revealed that the $\mathrm{CP}$ prevalence of children from trichorionic 
triplet pregnancies reduced to twins by selective termination (13.8 per 1000) was similar to that of children from trichorionic triplet pregnancies with no loss (18 per 1000), but the pregnancies with selective termination delivered at a later gestation. ${ }^{27}$ It is clear that we are in need for urgent reclassification of this controversy and it is author's belief that 4D sonography might help resolving it.

ART procedures have been regularly pointed out as one of the main reasons for the increasing incidence rates of $\mathrm{CP}$ in multiple pregnancies. After reports of increased risks of CP after IVF in a Swedish cohort study by Finnström and colleagues, authors found that there were no difference in type, severity of CP or background characteristics between children born after IVF and non-IVF children. The authors `interpretation is that etiological factors for CP do not differ, but that the increased prevalence of CP seen after IVF is due to high multiple rate and prematurity. ${ }^{28}$

\section{CONCLUSION}

Transvaginal 2D sonography is important modality in routine prenatal care because it allows accurate diagnosis and clinical management of multiple pregnancies during the first trimester. It was found that an early diagnosis of pregnancy number and placentation type (chorionicity and amnionicity) has positive influence on mortality and morbidity rate in multiple pregnancies.

Clearly 2D sonography is still the primary modality for diagnosing and evaluating multiple pregnancies, but due to its limitations an additional 3D sonography scan is recommended, because it provides more accurate and reliable diagnosis. 4D has several advantages over real-time $2 \mathrm{D}$ in the assessment of twin behavior. These include the capability of simultaneous visualization of both fetuses and assessment of their motor activity and most importantly of intertwin area. Therefore, for the time being, it should be considered as a method of choice for accurate diagnosis of isolated motor activity of single twins, and its true clinical value should be neither underestimated nor overestimated.

According to many reports multiple pregnancies compared to singelton have a 5-10 fold increased risk for $\mathrm{CP}$ and some of increased risk is due to the risk of preterm delivery, no mater whether it mono or dizygotic conception. In those cases CP is most probably complication of peripartal periventricular hemorrhage or leukomalacia. Known fact about twins is that the lower is the gestational age at the time of delivery, the probability that they are monozygotic is greater. Monochorionic multiple pregnancies and feto-fetal hemodynamic transfusion instability are leading to cerebral ischemia. For all of these reasons monozygotic multiple pregnancies have a greater risk of cerebral impairment.

Cerebral palsy in an apparently singleton pregnancy could be associated with an "vanishing" twin phenomenon. Some authors suggested that some congenital anomalies in apparently singleton pregnancies may be associated with the unrecognized or unrecorded loss of a co-twin.

According to many studies ART conceptions are at a greater risk of CP because of the greater risk of preterm delivery. There have been many attempts to minimize CP prevalence by reduction of number of fetuses in a multifetal ART pregnancy but without affect, most probably because the majority of ART conceptions are dizygotic.

\section{REFERENCES}

1. Vecek N, Kurjak A, Azumendi G. Fetal behaviour in multifetal pregnancies studied by 4D sonography. Ultrasound Rev Obstet Gynecol 2004;4:52-58.

2. Reinold E. Clinical value of fetal spontaneous movements in early pregnancy. J Perinat Med 1973;1:65-72.

3. de Vries JI, Visser GH, Prechtl HF. The emergence of fetal behaviour. I. Qualitative aspects. Early Hum Dev 1982;7:30122.

4. Hooker D. The Prenatal Origin of Behavior. Kansas: University of Kansas Press, 1952.

5. Arabin B, Bos R, Rijlardsdam R, et al. The onset of inter-human contacts. Longitudinal ultrasound observations in twin pregnancies. Ultrasound Obstet Gynecol 1996;8:166-73.

6. Reinold E.Clinical value of fetal spontaneous movements in early pregnancy. J Perinat Med 1973;1:65-72.

7. Prats P, Vecek N, Andonotopo W, Carrera JM, Kurjak A. Assessment of Multifetal Pregnancies. In Carrera JM, Kurjak A (Eds): Donald School Atlas of Clinical Application of Ultrasound in Obstetrics and Gynecology. Jaypee Brothers: New Delhi, 2006:133-64.

8. Vecek N, Solak M, Erceg-Ivkosic I. Four-dimensional sonography in multiple pregnancy. Gynecol Perinatol 2003;12:157.

9. Sadovsky E, Ohel G, Simon A. Ultrasonographical evaluation of the incidence of simultaneous and independent movements of twin fetuses. Gynecol Obstet Invest 1987;23:5-9.

10. Ferrari F, Cioni G, Prechtl HFR. Quantitative changes of general movements in preterm infants with brain lesions. Early Hum Dev 1990;23:193-97.

11. Kurjak A, Azumendi G, Prats P. The assessment of multifetal pregnancies by 3D/4D sonography. In Kurjak A, Azumendi G (Eds). The fetus in three-dimensions. Taylor and Francis: London, 2007;457-83.

12. Kurjak A, Vecek N. Three-Dimensional Sonography. In Blickstein I and Keith LG (Eds) Multiple Pregnancy 
Epidemiology, Gestation and Perinatal outcome Taylor and Francis:London 2005:309-21.

13. Dickey R, Taylor S, Peter YL, et.al. Spoontaneous reduction of multiple pregnancy: Incidence and effect on outcome. Am J Obstet Gynecol 2002;186:77-83.

14. Arabin B, Mohnhaupt A, van Eyck J. Intrauterin behavior of multiples. In Kurjak A (ed) Textbook of Perinatal Medicine. Vol II. London: Parthenon Publishig 1998;1506-31.

15. Piontelli A, Bocconi L, Kustermann A, et al. Patterns of evoked behavior in twin pregnancies during the first 22 weeks of gestation. Early Human Development.

16. Pharoah PO. Risk of cerebral palsy in multiple pregnancies. Obstet Gynecol Clin North Am Mar 2005;32(1):55-67.

17. Bonellie SR, Currie D and Chalmers J. Comparison of risk factors for cerebral palsy on twins and singletons. Developmental Medicine and Child Neurology 2005;47:587-91.

18. Pharoah POD. Cerebral Palsy and Multiple Births. In Blickstein I, Keith LG (Eds) Multiple Pregnancy. Taylor and Francis: London 2005;807-16.

19. Blickstein I. Cerebral palsy in multifetal pregnancies. Developmental Medicine and Child Neurology 2002;44:352-55.

20. Nelson KB, Grether JK. Causes of cerebral palsy. Current Opinion in Pediatrics 1999;11(6):487-91.

21. Pharoah PO, Adi Y. Consequences of in-utero death in a twin pregnancy. Lancet 2000;355:1597-602.
22. Scher AI, Patterson B, Blair E, et al. The risk of mortality or cerebral palsy in twins: a collaborative population-based study. Pediatr Res Nov 2002;52(5):671-81.

23. Landy HJ, Keith LG. The Vanishing Fetus. In Blickstein I and Keith LG ed. Multiple Pregnancy Epidemiology, Gestation and Perinatal outcome London. Taylor and Francis 2005:108-12.

24. Benson CB, Doubilet PM, Laks MP. Outcome of twin gestation following sonographic demonstration of two heart beats in the first trimester. Ultrasound Obstet Gynecol 1993;3:343-45.

25. Pharoah POD, Cooke RWI. A hypothesis for the aethiology of spastic cerebral palsy-the vanishing twin. Dev Med Child Neurol 1997;39:292-96.

26. Newton R, Casabonne D, Johnson A, et al. A Case-control Study of Vanishing Twin as a Risk Factor for Cerebral Palsy. Twin Research 2003;6(2):83-84.

27. Dimitrou G, Pharoah POD, Nicholaides KH, Greenough A. Cerebral palsy in triplet pregnancies with and without iatrogenic reduction. Eur J Pediatr 2004;163:449-51.

28. Finnström O, Karl-Gösta N, Otterblaed Olausson P. Cerebral palsy in children born after IVF in Sweden 1982-1995: Type of CP and maternal/obstetrical characteristics are similar to those in non-IVF children with CP. Acta Obstet Gynecol Scand 2005;84:1215-16. 